\title{
Bernard Juillerat. Une passion du sujet
}

Denis Monnerie et Pierre Lemonnier

\section{Q OpenEdition}

Journals

Édition électronique

URL : http://journals.openedition.org/jso/6175

DOI : 10.4000/jso.6175

ISSN : $1760-7256$

Éditeur

Société des océanistes

Édition imprimée

Date de publication : 15 décembre 2010

Pagination : $5-20$

ISBN : 978-2-85430-027-7

ISSN : 0300-953x

Référence électronique

Denis Monnerie et Pierre Lemonnier, «Bernard Juillerat. Une passion du sujet », Journal de la Société des Océanistes [En ligne], 130-131 | 2010, mis en ligne le 15 décembre 2010, consulté le 25 septembre 2020. URL : http://journals.openedition.org/jso/6175; DOI : https://doi.org/10.4000/jso.6175

(c) Tous droits réservés 


\title{
Bernard Juillerat. Une passion du sujet
}

\author{
par
}

Denis MONNERIE* et Pierre LEMONNIER**

«Une interprétation ou une théorie ne supporte le vieillissement que lorsqu'elle fournit simultanément les matériaux bruts qu'elle analyse. » (Juillerat, 1993 : 178)

De 1977 à 1982, Bernard Juillerat fut « secrétaire général adjoint responsable de la revue » au sein du bureau de la Société des Océanistes. Dixsept livraisons du présent Journal témoignent des week-ends et des soirées que Michèle Juillerat et lui consacrèrent à cette tâche aussi discrète qu'ingrate, qui va de la recherche d'articles à la transformation de manuscrits moins parfaits que ne le pensent leurs auteurs en des écrits « mis aux normes » d'une revue que l'on s'efforce de faire vivre. Manière de rappeler d'entrée la dette de la Société des Océanistes à l'égard de Bernard - et de présumer qu'à la différence de ces amis et collègues qui s'étonneront des quatre ans écoulés entre sa disparition et l'hommage que nous lui rendons ici, il nous aurait sans doute pardonné notre lenteur. Notre revue lui doit aussi un mémorable numéro spécial sur le chamanisme $\left(\mathrm{n}^{\mathrm{o}} 56-57,1977\right)$, pour lequel il sut réunir le concours de six éminents collègues étrangers. C'est à la même époque qu'il eut la générosité de donner de son temps et de sa patience pour animer au CNRS une " recherche coopérative sur programme » (RCP 587 AMOC, Anthropologie du monde océanien contemporain, 1980-1987) puis proposer un " groupement de recherche » (GDR ITSO, Identité et transformations des sociétés océaniennes, à partir de 1988) au sein desquels il réunit, pour la première fois, et pour le bien commun, des chercheurs comptant parmi les plus farouchement individualistes d'une profession qui n'en manque pas.

Parallèlement à cet engagement aussi désintéressé qu'efficace dans la vie de la recherche océaniste à un moment crucial de son institutionnalisation en France, Bernard Juillerat a produit une œuvre abondante, luxuriante même, forte de huit livres, auxquels s'ajoutent trois ouvrages dirigés ou co-dirigés, cinquante-deux articles et quarante-huit comptes-rendus qui, pour la plupart, constituent de brefs articles. Loin de se contenter d'aborder avec originalité la majorité des domaines de la discipline anthropologique, il a également renoué le dialogue avec la psychanalyse, tant de manière théorique qu'à travers des études de cas minutieusement construites et analysées. Partiellement écrite (1992) ou traduite en anglais (voir la bibliographie établie par Isabelle Leblic pour ce volume), son œuvre a fait l'objet de multiples commentaires, à la fois sous forme de recensions et lors des débats qu'il a initiés pour exposer et défendre systématiquement les hypothèses ou points de vue qui étaient les siens. C'est ce dialogue, d'autant plus dense et précis qu'il était proposé par un chercheur que seule sa passion de la science faisait sortir de sa réserve naturelle, que poursuivent la vingtaine de chercheurs qui lui rendent ici hommage.

\section{Les multiples facettes d'un chercheur accompli}

Initialement formé à Lausanne aux lettres et aux langues (anglais et espagnol), puis à l'anthropologie, auprès de Roger Bastide, à la

* Professeur à l'Université de Strabourg, monnerie@umb.u-strasbg.fr

** Directeur de recherche au CNRS CREDO, pierre.lemonnier@univ-provence.fr 
Sorbonne, Bernard Juillerat était d'abord un homme de terrain et un praticien de l'enquête monographique sous toutes ses formes. Ayant découvert l'Afrique alors qu'il était affecté à une école suisse de Kinshasa, il réalise entre 1966 et 1968 une première enquête de seize mois chez les montagnards Mouktélé du Nord Cameroun, avec l'aide du Fonds national de la recherche scientifique suisse. Il soutient à Paris en 1969 une thèse de doctorat d'ethnologie consacrée à l'organisation sociale des Mouktélé qui sera publiée en 1971 sous le titre Les bases de l'organisation sociale chez les Mouktélé (Nordcameroun). Structures lignagières et mariage. C'est comme africaniste qu'il a rejoint le CNRS au début des années 1970. Il s'est ensuite rendu chez les Yafar, un peuple de langue amanab du Haut Sépik de Nouvelle-Guinée, sur les conseils d'Alfred Gell qui menait alors ses propres travaux chez les Umeda voisins, dans les Border Mountains, dans l'extrême ouest de ce qui n'était pas encore l'État indépendant de Papouasie Nouvelle-Guinée (Juillerat, 1997 'Yangis, Lacan...' : 36). Il devait y effectuer cinq séjours entre 1970 et 1986. Il se rendit ensuite chez les Bánaro du Bas Sépik (en 1989-1990), afin d'évaluer le travail pionnier effectué par Richard Thurnwald en 1913 et 1915 à propos de cette société dans laquelle le grand ethnologue allemand n'avait pourtant pas résidé ainsi que pour étudier une société très différente des Yafar, mais sans doute parce que Thurnwald avait lui aussi un intérêt pour les phénomènes psychologiques, comme le signale Marion Melk-Koch dans ce volume. Dans les années 1990, il effectua plusieurs missions en Espagne, mais sans retrouver la passion pour le terrain qu'il avait connue en Afrique et en Nouvelle-Guinée.

La variété des thèmes abordés par Bernard Juillerat indique qu'il était de ces anthropologues pour qui la démarche monographique s'impose comme premier horizon d'enquête, dès lors qu'on ne sait a priori où se nichent les aspects les plus spécifiques d'une société donnée. De la technologie de l'essartage (1983) aux transes (1975), de l'étude des couleurs (1978) à l'analyse de la parenté (1977) et de l'histoire du travail en plantation (1979) à l'ethnomusicologie (1993), en passant par l'ethnographie et la théorisation des relations entre mythe et rite (1991) ou par l'étude des rapports de production, aucune question anthropologique n'était étrangère à Bernard. Tout au long de sa carrière, il a par ailleurs tiré parti de tout l'éventail des outils d'observation disponibles : photographie, enre- gistrement sonore, cinéma. À ses écrits, ce passionné de cinéma épris de photographie a ajouté trois films en $16 \mathrm{~mm}$ - un sur l'Afrique et deux sur les Yafar -, plusieurs centaines de photographies $^{1}$ et des collections d'objets désormais conservés à la photothèque et dans les réserves du musée du quai Branly ainsi qu'au National Museum and Art Gallery de Port Moresby évoquées plus loin par Nicolas Garnier. Et il y eut longtemps une vitrine « Groupe linguistique des Amanab» au musée de l'Homme (voir photo dans Jeudy-Ballini et Juillerat, $2002: 25$ ).

Parallèlement à sa connaissance de l'histoire de la discipline et des dossiers anthropologiques les plus récents, qui se lit constamment en filigrane de ses propositions théoriques et qui s'explicite dans ses comptes rendus d'ouvrages, Bernard Juillerat a réalisé une ethnographie qui, dans tous les domaines, fut minutieuse. Dans la somme qu'il a consacrée aux Yafar - Les enfants $d u$ sang (1986) -, il a utilisé ses vastes connaissances ethnographiques et théoriques pour montrer avec succès, comment plusieurs points de vue (et divers domaines de la vie), imaginaires autant que matériels, se renvoient les uns aux autres dans la vie quotidienne et rituelle d'un groupe humain. La rigueur et la recherche de l'exhaustivité se lisent dans les domaines les plus variés : plans de maisons et termes architecturaux $(1986: 113,116)$; exemples de toponymes (1986:151) ; inventaire des groupes domestiques (1986: 118); horticulture, chasse et élevage (1986: 155-222); pérégrinations des Yafar (1986: 38-45); plans de hameaux (1986: 49-53); échanges intertribaux (1986 : 226-234); analyse du mariage à partir d'une étude de cas réels (1986 : 286-328) ; accusations de sorcellerie (1986 : 453-467), etc.

$\mathrm{Au}$-delà de cette très complète monographie, les écrits de Bernard recèlent nombre de descriptions ethnographiques dont la finesse - la délicatesse même - charme le lecteur, en même temps qu'elle comble sa curiosité ; au hasard : l'ethnolinguistique d'une formule magique (1995: 67-85) ou le récit de la mise à l'écart d'un malade (1997 : 78-79). Quiconque a pratiqué l'enquête de terrain peut s'identifier à lui, tout stupéfait de rendre son salut militaire à un Yafar embringué dans une aventure millénariste, et ressentir l'automatisme d'un geste dû à la fatigue, où se mêlent une pointe d'humour et l'abandon d'un moment à l'absurde de la situation (1997: 71). Et ceux qui ont redouté une chute ou l'invisible présence d'un serpent tout en crapahutant dans un jardin papou se voient, comme Bernard, 
chercher du pied la surface ferme et rassurante du sol dissimulée sous le fouillis végétal du lieu (1999 : 196).

C'est sur cette ethnographie méticuleuse associée à une sensibilité vigilante que Bernard Juillerat a fondé les développements théoriques qui ont construit sa renommée internationale. Il a rappelé de la plus belle manière que la démarche - la découverte - anthropologique est une quête de longue durée, faite d'allers-retours entre « terrain » et « théorie ». Aux thèmes multiples de ses recherches, les articles ici rassemblés en son hommage font écho, puisque chacune à sa façon, les dix-sept contributions du présent volume abordent un ou plusieurs des domaines sur lesquels a porté la réflexion de Bernard Juillerat.

Adepte du comparatisme, attentif à la théorisation, Bernard Juillerat fut un homme de débat, tant pour proposer ses idées et interprétations aux commentaires de ses collègues que pour s'élever contre des positions qui lui semblaient peu établies, douteuses voire dangereuses. Nous rappellerons ici trois des discussions que Bernard a marquées de ses interventions, en l'occurrence, trois thèmes par lesquels les mélanésianistes ont alimenté la réflexion anthropologique générale de ces trente dernières années : la question du rituel, du sens et de l'exégèse ; celle du sujet et de l'anthropologie psychanalytique; et celle des rapports avecl'histoire.

\section{Une anthropologie du rituel}

Outre Naven (Bateson, 1936), régulièrement commenté et ré-analysé ( $c f$. Houseman et Severi, 1994 ; Silverman, 2001 pour une bibliographie récente) - y compris, d'ailleurs, par Juillerat (1999, 2001 chapitre 9, prenant en compte les découvertes récentes de Silverman), ou encore ici même, par Christian Kaufmann, de nombreux rituels mélanésiens ont plus ou moins récemment fait l'objet de descriptions et d'analyses qui ont marqué la discipline. Pour la seule NouvelleGuinée, citons les rites mortuaires des Daribi (Wagner, 1973), l'analyse des cérémonies funèbres des Kaluli (Schieffelin, 1976 ; Feld, 1979), les représentations funéraires de la dé-conception chez les Mekeo (Mosko, 1983), l'étude comparatiste des funérailles dans le Massim (Damon et Wagner, 1989), l'étude du culte Ida des Umeda voisins des Yafar (Gell, 1975), les recherches sur les cultes millénaristes de Madang (Lawrence, 1964) ou des Baining
(Whitehouse, 1995), les analyses des cultes de fertilité des Mountain Ok (Barth, 1975, 1987), ou encore les nombreux travaux consacrés aux cultes masculins : ceux des Arapesh (Tuzin, 1980, 1997), des Gnau (Lewis, 1980), des Sambia (Herdt, 1981, 1987, 2003) et des Baruya (Godelier, 1982). Avec pas moins de trois livres (Edipe chasseur, 1991 ; Shooting the Sun, 1992 ; L'avènement du père, 1995) et une série de débats dans la section Correspondence de Man en 1980-1981 et 1990, les écrits de Bernard Juillerat tiennent une place de choix au palmarès de ces travaux qui ont chacun apporté un regard particulier et des développements théoriques nouveaux sur le rituel. D'une part, du fait de son interprétation psychanalytique des rites Gungwan et Yangis - sur laquelle on reviendra plus loin-, mais aussi par sa participation à la controverse plus générale sur le sens des " religions mélanésiennes » et leur exégèse déclenchée par les commentaires de Ron Brunton (1980) sur l'interprétation par Gell du rite Ida des Umeda, très proche de Yangis.

Dans un article de Man où il corrélait l'" ordre » (au sens de cohérence) relatif de divers systèmes religieux mélanésiens avec leur place dans les stratégies politiques des groupes et des individus, Ron Brunton a reproché aux anthropologues de surévaluer ce degré d'homogénéité ${ }^{\text {, alors }}$ même qu'il varie selon les sociétés considérées. Bien que considérant finalement le rite de fertilité Ida des Umeda comme " possédant un considérable degré de cohérence », Ron Brunton en présenta l'analyse par Alfred Gell (1975) comme un exemple de ces travers " des » anthropologues sans autre précision - dont les postmodernes devaient faire ensuite leur miel : ne tenir compte ni des difficultés qu'ils ont à obtenir des informations sur les systèmes religieux ni du désintérêt éventuel de leurs informateurs pour ces questions ; conjecturer l'existence d'un noyau dur de croyances et supposer que les membres d'une société y voient eux-mêmes un ordre ; ignorer les éléments incohérents d'un système de sens, les opinions divergentes selon les informateurs et les contradictions chez un même informateur; n'expliquer ni l'abandon de certains épisodes rituels nil'adoption d'éléments nouveaux dénués de sens ; etc.

Selon Ron Brunton, l'étude d'Alfred Gell était de surcroît représentative de ces «modèles extérieurs » d'inspiration structuraliste construits par l'anthropologue en l'absence de toute exégèse susceptible de guider ses pas (Brunton,

2. Rappelons que cet order dépend, selon Ron Brunton :1) du degré d'élaboration et de l'ampleur d'un système religieux ; 2) de sa cohérence interne ; 3) de l'uniformité des croyances et des pratiques d'un individu à un autre ;4) de leur persistance dans le temps (Brunton, $1980: 122$ ). 
1980 : 117). Fort de son expérience du culte Yangis, qu'il avait observé en 1976, et des heures de commentaires qu'il venait de recueillir et d'analyser chez les Yafar, Bernard Juillerat fit valoir son point de vue, à la fois contre celui, sans nuance aucune, de Ron Brunton, et contre celui d'Alfred Gell pour qui le même rituel ne suscitait aucun commentaire de la part des Umeda, dans une société pourtant de langue voisine, située à quatre heures de marche des Yafar. Pour Bernard, le « désintérêt » apparent des informateurs pour le sens des rituels est à mettre en relation avec leur souci de préserver un secret tel que, selon les Yafar, «tous les hommes mourraient» si les femmes venaient à le connaître. La parcellisation de ce savoir n'illustre aucune incohérence, mais au contraire une caractéristique de systèmes de sens dont les éléments sont pensés comme devant être mis en relation par les acteurs eux-mêmes. Bernard Juillerat rappelait également que ce savoir secret qui lui fut confié sur le terrain fait l'objet d'une transmission institutionnalisée entre des hommes appartenant aux deux moitiés chargées de tâches complémentaires dans Yangis. Au-delà de la controverse sur l'existence d'une exégèse à propos de Ida/Yangis, il insistait sur les insuffisances des "propriétés miraculeuses de l'analyse structurale » reconstruite « à la maison » par Gell. À quoi sert, par exemple, demandait Bernard Juillerat, de souligner la couleur rouge du corps des danseurs ipele si on ignore que le mythe signale que c'est la couleur du sang de l'hémorragie qui tua leur mère à leur naissance $(1980: 733)$ ?

Irrité par l'absence de nuance des propos respectifs d'Alfred Gell et de Ron Brunton, il parla des « erreurs d'interprétation » commises par le premier et attribua le continuum d'homogénéité relative des systèmes religieux mélanésiens proposé par Ron Brunton aux différents niveaux de savoir ethnographique des anthropologues! (1980 : 734). Pour sa part, Alfred Gell opposa sa propre approche " sociologique " - traitant de mariage, du cycle de vie, du leadership villageois - et, partant, reliée à la théorie anthropologique générale, à celle de Bernard Juillerat, supposée enfermée dans une explication du rituel par des aspects du mythe et « laissant de côté toute question analytique intéressante » (Gell, 1980 : 736).

En dépit des échanges d'amabilités qui émaillent cette correspondence, Bernard Juillerat convia par la suite Alfred Gell et huit autres collègues à commenter sa propre interprétation de ce rituel pour en débattre dans un livre unique en son genre - Shooting the Sun (1992). Dans sa lettre à Man, il n'avait fait qu'indiquer combien la prise en compte de la glose, du secret et de l'empilement ou de la mise en relation des savoirs changerait la vision anthropologique de Idal Yangis. Dans l'ouvrage collectif dont il prit l'initiative, il présentait pour la première fois son interprétation psychanalytique de Yangis. Pour lui, ce rite est la mise en scène d'une histoire œdipienne mythique, secrète. Il s'agit de la théâtralisation d'aspects fondamentaux de la constitution du cosmos où la figure maternelle est présente à la fois comme terre-mère et dans le soleil qui est le sein maternel. À la fin de Yangis, en une séquence cruciale bien que ne durant que quelques minutes, les archers décochent leurs flèches vers le soleil après les avoir d'abord pointées vers le sol, donnant à voir cet aspect important des significations du rite. Yangis possède simultanément un plan totémique mettant en scène la naissance de l'humanité et de la société à partir des deux espèces caractérisant les moitiés de la société Yafar et qui sont les facettes totémiques masculine et féminine de l'Humain dans ses processus de reproduction et de socialisation. Enfin, Bernard Juillerat insiste sur le fait que « le processus émotionnel fondateur qui est à l'origine du rituel doit être distingué de l'émotion induite en retour [par le rituel] sur l'individu » (1992 : 111).

Surtout, dans Shooting the Sun, la multiplicité des approches proposées par les différents auteurs constituait en soi une réflexion théorique et méthodologique sur l'étude et l'exégèse du rituel en général, y compris sous la forme d'une série d'interprétations selon les approches favorites de chacun "plus complémentaires qu'exclusives les unes des autres» (1992: 285). Elle soulevait également la question des rapports entre des faits universels de constitution et de structuration de la personne et des institutions. En la matière, l'apport principal de Bernard Juillerat fut de mettre en avant la question du sens du rituel pour les sujets membres d'une société donnée, au moment où elle commençait d'être d'emblée évacuée des recherches sur le rituel. Pour une majorité de spécialistes du rituel, le problème est d'expliquer en quoi il est un type d'action particulier. Si celui-ci « dit» quelque chose, lit-on souvent désormais, il ne serait qu' « un accessoire redondant de la règle sociale » (Houseman et Severi, $1994: 164$ ), mais rien n'indique en quoi il est une façon spécifique et nécessaire d'exprimer, interpréter, ou « moduler » (Gell, in Juillerat, 1992 : 142) celle-ci. Les recherches en anthropologie cognitive, en particulier, considèrent comme démontrée, sans autre forme de procès, l'inutilité de toute recherche de la signification d'un rituel, tant pour les sujets qui le vivent que pour les anthropologues qui 
tentent de comprendre sa place dans un système de sens (par exemple Humphrey et Laidlaw, 1994 : 192, 262). Sans autre forme de procès, c'est-à-dire comme si ce débat relancé par Bernard Juillerat n'avait pas existé. On comprend qu'il ait complété ses multiples travaux sur la prise en compte du sujet et le rapprochement entre psychanalyse et anthropologie par une critique exaspérée des prétentions et des raccourcis de l'anthropologie cognitive (2001: 9-38).

Comme l'écrit ici André Green à propos de l'implication de Bernard Juillerat dans l'approche psychanalytique, celui-ci ne faisait pas les choses à moitié : il «n'était pas de ceux qui se contentent de quelques citations de Freud pour conclure rapidement à la pertinence d'une référence à la psychanalyse pour l'anthropologie ". C'est la genèse de son approche d'anthropologie psychanalytique que nous voudrions retracer à grands traits à partir de ses publications, dans les pages qui suivent.

\section{De l'attention au psychique à l'anthropologie psychanalytique}

«Pionnier de l'anthropologie psychanalytique [...] Géza Roheim [...] s'est toujours présenté comme le seul ou le premier ethnologue qui ait adhéré totalement à la psychanalyse. » (Dadoun, in Roheim, 1967 : 9)

Entre 1923 et 1931, Géza Roheim « fit du pays Somali, de l'Australie centrale [Pitjentara], de l'île Normanby [en Nouvelle-Guinée] et des Indiens Yuma de l'Arizona son champ de recherche » (ibid.). Autre pionnier de l'anthropologie psychanalytique, Georges Devereux (1908-1985) travailla sur les Sedang Moï du Vietnam et les Indiens Mohave du sud-ouest des États-Unis. L'influence de ce dernier se fit particulièrement sentir en France dans les années 1960 et 1970 à travers son séminaire d'ethnopsychiatrie de l'École pratique des hautes études, passage quasi obligé pour une grande partie d'une génération d'anthropologues - dont beaucoup d'ailleurs ne s'orienteront pas nécessairement vers l'anthropologie psychanalytique.

Le parcours initial de Bernard Juillerat ne semble pas se dérouler dans une continuité directe à ces deux grands pionniers - qu'il citera ensuite dans nombre de ses textes. Cependant, le lien avec Devereux se fait à travers Roger Bastide, qui fut son directeur de thèse. En l'absence d'entretiens publiés donnant une vision argumentée par lui des raisons de son orientation psychanalytique comme aboutissement théorique de ses recherches en Océanie, l'étude d'un choix de publications dans les pages qui suivent, considérées comme des jalons significatifs de ce développement de l'œuvre, servira de guide pour une tentative de compréhension de ce parcours scientifique, de ce chemin qui mena Bernard Juillerat vers l'anthropologie psychanalytique.

Intitulé Les bases de l'organisation sociale chez les Mouktélé (Nord-Cameroun). Structures lignagères et mariage, l'ouvrage issu de sa thèse, publié en 1971, ne laisse guère présager de l'évolution future de Juillerat. C'est un travail classique, bien délimité en son titre, dont l'écriture claire et précise donne le ton de l'ensemble des textes à venir. Elle-même spécialiste du Cameroun, Jeanne-Françoise Vincent nous présente dans ce numéro les travaux de Juillerat dans ce pays. La conclusion est resserrée sur la problématique de départ :

« [le] problème lignager [a été traité] sous deux plans distincts, bien que liés entre eux. Le premier, essentiellement diachronique, a permis de saisir le processus historique par lequel [...] les patrilignages se sont constitués et se sont scindés [...] le second plan, plus synchronique, nous a conduit à un tableau général de l'organisation segmentaire [...] le groupement de plusieurs [segments] tshay en une unité plus vaste se fait toujours par le truchement du territoire. » (Juillerat, 1971 : 243-244)

Dans cette étude d'organisation sociale, les conclusions sur les relations d'affinité entrouvrent un peu la porte à des considérations de relations inter-personnelles.

«Dans le cadre du régime matrimonial, les aspects les plus marquants sont l'importance occupée par la parenté cognatique dans le régime exogamique et l'instabilité du mariage [...] [pour celui-ci on peut] distinguer la séparation due à des raisons externes et la séparation due à des causes internes, inhérentes au couple même (incompatibilité d'humeur) [...] seul le second type de séparation [entraîne] une rupture définitive du mariage, tandis que le premier type [n'occasionne] le plus souvent qu'une séparation temporaire. » (Juillerat, $1971: 244$ )

On trouve, dans le cours même de cette étude monographique, d'autres ouvertures sur l'étude fine des relations inter-personnelles.

Dans le cadre de l'étude des processus qu'il nomme du plaisant néologisme de courtisation désignant «l'ensemble des comportements amoureux prémaritaux » (1971: 153) -, l'intérêt de Juillerat pour les langues et le langage, jamais démenti depuis ses études à Lausanne jusqu'à ses dernières publications, se combine à une attention, non aux « comportements psychologiques individuels, mais seulement [aux] conventions que la société a créées pour résoudre la difficulté [...] [car] la courtisation suppose un problème de 
communication entre individus placés dans un contexte psychologique particulier [...] Problème de communication, donc principalement de langage » (ibid. : 156). Cette mise en perspective n'entame en rien la finesse de quelques rares touches psychologiques disséminées dans les pages consacrées à la courtisation (153161).

« Cette grande réserve dans les comportements de langage [pour la courtisation en face à face] peut parâ̂tre contredire ce que nous avons dit [...] concernant les attitudes des groupes de jeunes sur les marchés et la facilité avec laquelle s'y nouent des intrigues. Nous pensons à ce propos qu'il faut distinguer les situations individuelles et les situations de groupe. Les conditions psychologiques et sociales dans lesquelles se trouve le jeune homme isolé à la recherche d'une [amie] mô $D a$ sont tout autres que celles du même individu incorporé à un groupe. Dans ce dernier cas, la bière de mil modifie en outre profondément le psychisme du groupe. Les intrigues qui en résultent ne sont souvent que passagères. Le mode de courtisation "en groupe" basé sur la compétition, le divertissement, la dépense ostentatoire et l'ivresse peut être considéré comme une forme d'affranchissement des contraintes que suppose le mode de courtisation individuel. À la circonspection et au tact dont est empreint le comportement de l'amant isolé s'opposent le franc-jeu et la quasi brutalité du comportement collectif. » (ibid. : 160)

Les plus belles lignes de cette étude de la courtisation sont sans doute celles où sont décrites les rencontres chez la jeune fille, avec cet exemple de dialogue de libertinage conventionnel :

[Lui :] « Lève-toi, sœur, et verse-moi de l'eau » [Elle : ] « Est-ce le diable qui me réveille ainsi ?»

[Lui : ] «C'est moi, lève-toi et donne-moi de l'eau, te dis-je. Mon âme t'a suivie depuis longtemps déjà ; c'est pourquoi je suis venu aujourd'hui. Vais-je la trouver chez toi ? Mes yeux te voient belle et mon esprit ne sait que faire, ma sœur [...] » (ibid. : 157)

Ainsi, dans cette étude serrée des relations sociales, d'où n'émergent à propos de relations inter-personnelles que quelques réflexions sur la psychologie des acteurs sociaux, rien ou presque ne permet d'envisager la voie que va plus tard privilégier Bernard Juillerat. Il faudra un nouveau terrain, océaniste, pour favoriser, très progressivement nous allons le voir, son orientation vers l'anthropologie psychanalytique. Après ces premiers travaux sur les Mouktélé viennent donc les recherches en Papouasie Nouvelle-Guinée, dans le Sépik Occidental au nord des Border Mountains.

L'étude par Bernard Juillerat de la possession chez les Yafar intitulée "Transe et langage en Nouvelle-Guinée », fut publiée en deux parties dans le JSO (1975a et b). Il y porte son attention sur des phénomènes de transe, aussi qualifiés de crise, comportement hystérique ou dissociation et décrits comme des symptômes, ou un syndrome. Les deux articles présentent une étude de l'insertion dans l'institution et de la récupération par la culture de phénomènes qu'il qualifie en leur état initial de "sauvages ", sans doute au sens de Claude Lévi-Strauss (1962a). Plus précisément il étudie la « socialisation et [la] symbolisation de quelques symptômes. Mon propos est de suivre plus particulièrement ici quelques-uns des symptômes de la dissociation dans leur processus de récupération par la culture » (1975b : 388). Le premier article est consacré à la " possession médiumnique » chez les Yafar. La passion du terrain, qui fut - avec un goût pour les voyages - l'une des assises de la vocation anthropologique de Juillerat (Michèle Juillerat, communication personnelle), y est illustrée par ses qualités d'ethnographe, déjà manifestes dans ses travaux chez les Mouktélé et qui se trouvent encore plus en évidence dans la description très fine et complète qu'il donne des transes observées à Yafar. À nouveau, le langage est une composante centrale de son étude et de sa réflexion.

«Outre un timbre et des intonations enfantines, le médium utilise un vocabulaire spécial d'une cinquantaine de termes qui, se mêlant au lexique habituel, correspondent à des signifiés parmi les plus courants : aliments, animaux, positions parentales [...] cette langue des dieux (nabasa na mô)... n'est donc pas une langue secrète, [elle] n'a pas pour fonction de susciter un ésotérisme d'où les femmes seraient exclues, mais seulement de démarquer la présence divine par rapport au contexte humain et d'authentifier la possession et la fonction médiumniques. Ce lexique spécial, connu de tous, est aussi bien utilisé par les interlocuteurs humains que par les dieux eux-mêmes. » (1975a : 208)

Le second article a une portée comparatiste, resituant « le cas amanab [Yafar] dans le cadre plus large des phénomènes de dissociation ou de transe rituelle observés en Nouvelle-Guinée » (1975b : 379).

«[Les] échantillons [de la comparaison] ont été choisis dans la mesure où ils illustrent chacun un stade différent dans le processus de contrôle progressif de la dépersonnalisation et où ils constituent une échelle [...] postulant un changement qui ne se ferait pas au hasard, mais qui, partant du syndrome "sauvage", tendrait dès le départ vers une récupération croissante de ce syndrome par la culture. Le processus de socialisation et de ritualisation est conçu comme contingent [...] chaque société orientera le syndrome vers un domaine culturel différent (en fonction de ses préoccu- 
pations) ou l'intégrera peut-être dans un rituel religieux préexistant. » (1975b : 379-380)

La synthèse de Bernard Juillerat montre que la surdité, un sentiment de refroidissement, l'agressivité et - largement développé dans sa présentation - un rapport très particulier au langage, sont les symptômes principaux de ces dissociations.

«Selon ce qu'elle entend en faire, la société remodèle le syndrome en donnant plus ou moins de place à chacun des symptômes et en les dotant d'un sens » (ibid. : 392)

Ce « sens » toutefois n'est pas, directement au moins, celui de la langue. Nous aimerions en effet souligner ce qu'il considère comme " un phénomène constant » : l'association de la crise avec une utilisation de la parole dans laquelle « le sujet évite de parler la langue de son groupe» (ibid. : 391). Bernard pointe ici un phénomène largement répandu en Océanie, des usages spécifiques de la parole étroitement associés à des formes, spécifiques elles aussi, de sociabilité.

Pour les sources comparatistes de ces articles, les études ethnographiques sur la NouvelleGuinée et l'Océanie sont privilégiées. Sur la quarantaine d'auteurs cités, moins d'une dizaine le sont pour des ouvrages généralistes de psychiatrie ou de psychanalyse - trois d'entre eux écrits par des ethnologues, Roger Bastide, Georges Devereux et Ioan Lewis. L'attention de Juillerat aux approches psychiatrique et psychanalytique des phénomènes de dissociation se porte ici surtout sur des synergies déjà établies entre l'ethnologie et ces deux disciplines. Ainsi, il reprend le dialogue entre l'anthropologue Louis Langness et les psychiatres Steven Hirsch et Marc Hollender à propos de la définition, puis du réajustement catégoriel, des crises negi negi des Bena Bena. D'abord qualifiées par Langness de « psychose hystérique», elles deviendront des « pseudo-psychoses », à l'incitation d'une publication ultérieure de Hirsch et Hollender (Juillerat, 1975b: 383-385). Juillerat poursuit la réflexion de Langness pour l'étendre au syndrome féminin genefafaili.

« Personnellement, et pour autant qu'on puisse en juger d'après la documentation succincte qui nous est donnée, il me semble très risqué de parler de folie pour un syndrome totalement ritualisé et intégré au contexte funéraire. Le caractère auto-induit de l'attaque genefafaili, très proche semble-t-il de la transe des médiums amanab [Yafar], paraît ici évident. " (ibid. : 385)

Il n'en reste pas moins que, dans ces deux articles, c'est surtout dans les notes de bas de page que Juillerat développe ses idées en relation à la psychiatrie et la psychanalyse. Ainsi la note 14 (ibid. : 386-387), où il réagit à une proposition par Mary Reay de judiciarisation répressive du comportement de l'homme komugl des Kuma. La réaction de Juillerat est au diapason des idées de contestation de la psychiatrie, assez répandues au plan mondial dans les années 1960 et au début des années 1970 :

«pourquoi [...] vouloir imposer à ces sociétés un système répressif propre à l'Occident (où il est d'ailleurs de plus en plus contesté), tandis qu'elles ont précisément le moyen d'assimiler le syndrome sans le condamner et d'intégrer le "psychotique" sans l'isoler?» (ibid.)

Alors même que l'attention au psychique y est centrale, l'approche de ces deux articles diffère sensiblement de celle, plus proprement psychanalytique, qui deviendra sienne un peu plus d'une décennie plus tard à partir des Enfants $d u$ sang. Pour comprendre les différences, on se réfèrera à la synthèse que Juillerat propose des textes de 1975 dans laquelle on peut percevoir l'influence des travaux de Bastide sur la façon dont Juillerat traite des transes en NouvelleGuinée.

« Si l'on élargit le problème en ne plaçant plus le syndrome à l'origine du processus de réponses complémentaires, mais en l'incluant au centre d'un complexe plus vaste qui serait celui de la vie et de l'histoire sociale du groupe, il faut alors concevoir la crise initiale, "sauvage", comme un accident, une "mauvaise réponse" ou une sorte de "corps étranger" que l'organisme social chercherait ensuite à identifier et à neutraliser. Ce processus d'identification-assimilation peut être considéré comme le résultat d'un réflexe de défense du corps social contre le danger que représente le syndrome en tant qu'élément allogène. La société cherche à vivre avec son syndrome en le mettant en circuit ou pour le moins en le plaçant "sur orbite" et en lui laissant ainsi sa marginalité sans le rejeter. » (1975b : 393)

Proposons de voir en ces lignes plus l'influence des travaux de certains psychiatres - et antipsychiatres - que celle, majeure, de la psychanalyse qui va ensuite marquer la recherche sur les Yafar, et en particulier sur leurs mythes et le rituel Yangis. L'incertitude des notions qui apparaît dans l'usage de guillemets va, dans la suite de l'œuvre, laisser la place à l'usage de concepts établis par la psychanalyse. Surtout, à cette première approche où la psychanalyse contribue à éclairer la transe, phénomène décrit comme étant de l'ordre du syndrome et au fond marginal, va succéder une démarche beaucoup plus ambitieuse où la psychanalyse occupe une 
place désormais centrale puisqu'elle sera mobilisée pour interroger les tréfonds "infrasociaux » de l'organisation et de la formation de traits fondamentaux de la société et de la culture yafar.

Après une longue série d'articles sur les Yafar aux sujets très divers (voir la bibliographie), la parution en 1986 de Les enfants du sang : société, reproduction et imaginaire (1986), monographie très complète et détaillée, fonde la problématisation de l'ensemble de l'ethnographie constituée par Juillerat. Dans une conversation, il disait s'être orienté vers la psychanalyse en raison du caractère œdipien des mythes yafar (en 2000 ; Agnès Clerc-Renaud, communication personnelle) ce que confirment les lettres à Philippe Peltier publiées dans ce numéro. Dans Les enfants $d u$ sang, on le voit se tourner vers la psychanalyse, posant de nombreux jalons ethnographiques et interrogations ouvrant sur cette approche. À propos des mythes, mais aussi du non-dit yafar entourant la reproduction et la figure maternelle en particulier et de nombreux autres aspects de la société. Dès l'introduction, remarquant qu'il existe "d'excellentes et rares recherches d'ethnopsychanalyse », il souligne aussi que :

«quelques psychanalystes anthropologues s'obstinent à négliger les facteurs sociaux et historiques, alors que les spécialistes de la parenté ou du pouvoir ne disent mot des représentations psychiques; d'autres encore entendent tout expliquer à partir d'une nébuleuse postjungienne où l'homme, détaché de tout contexte matériel, est réduit à son imaginaire. » (1986 : 10)

Au fil de l'ouvrage, Juillerat cite Gérard Mendel, Erich Fromm, André Green, Julia Kristeva, Roger Bastide - mais aussi l'inclassable Gaston Bachelard. Ainsi, dans le cadre de cette monographie classique, la réflexion à partir de la psychanalyse progresse par touches discrètes mais fermement appliquées, de façon cumulative. La brève conclusion met en place les lignes de force de son programme d'analyse du monde yafar. L'influence de Bastide y est explicite.

« [Ce dernier] a montré qu'une psychanalyse de la culture n'équivalait nullement à une réduction du social au psychique, mais demandait de dégager les "lois de transformation" qui ont permis à la structure psychique de se reproduire dans la structure socioculturelle. En ce qui concerne les Yafar, on a vu à quel point l'ensemble conceptuel construit à partir des structures infrasociales est récurrent à de multiples niveaux du système. [...] Structures de groupes, systèmes d'alliance, formes de "l'initiation" des garçons, essaimage des clans, transmission des droits fonciers, rituels de protection de la personne ou de reproduc- tion des unités totémiques, etc. : tous ces dispositifs font référence à la filiation (agnatique institutionnelle ou utérine), à la relation à la mère, réelle ou mythique, à la transgression œdipienne et à sa régulation, à l'inversibilité temporelle de l'ordre social, au rapport transcendant avec les 'sangs' masculins ou féminin. » $(1986: 514)$

La perspective, comme l'ambition théorique, s'affirment ici bien plus larges et fondamentales que dans les études sur la transe. Il ne s'agit plus seulement d'une réflexion sur l'intégration, ou la « mise sur orbite » d'un syndrome personnel de dissociation par les sociétés et les cultures. Ayant appréhendé que, de façon généralisée, l'ensemble conceptuel yafar est élaboré à partir de structures psychiques infrasociales, Juillerat entend mettre au jour des lois par lesquelles la structure psychique se reproduirait dans la structure socioculturelle. Plus tard, il écrira que :

« l'anthropologie peut être qualifiée de psychanalytique quand l'utilisation de concepts freudiens ouvre de nouvelles voies à l'interprétation des faits sociaux et des contenus culturels. C'est le cas lorsque les fonctions et représentations psychiques opérant chez la plupart des individus, tout particulièrement celles qui sont inconscientes, se traduisent par des productions collectives. » (2004: 158)

Le programme psychanalytique de Juillerat annoncé dans Les enfants du sang est mis en œuvre dès 1988, avec un article intitulé «"Une odeur d'homme" : évolutionnisme mélanésien et mythologie anthropologique à propos du matriarcat » (repris dans Penser l'imaginaire), qui sera suivi de bien d'autres, et de quatre livres où culminent cette réflexion trop tôt interrompue. C'est d'abord, en 1991, Edipe chasseur : une mythologie du sujet en Nouvelle-Guinée, préfacé par le psychanalyste André Green. Puis Shooting the Sun. Suit, en 1995, L'avènement du père. Rite, représentation, fantasme dans un culte mélanésien qui présente une description détaillée du rite Yangis des Yafar et son analyse en termes psychanalytiques. Puis, en 2001, Penser l'imaginaire, qui rassemble des Essais d'anthropologie psychanalytique, dont certains d'humeur polémique, comme celui, inédit, ouvrant l'ouvrage, qui traite de façon très incisive de « La dérive cognitiviste en anthropologie ». Confronter son approche psychanalytique à d'autres terrains (Juillerat, Bidou et Galinier, 2005) et à d'autres démarches anthropologiques qu'il considère comme scientifiquement pertinentes et fondées sur une ethnographie fiable et détaillée a été l'une des préoccupations de Juillerat. On trouvera dans ses nombreux comptes-rendus de lecture de multiples suggestions dans ce sens. 
Mais c'est avec la publication, en 1992, de Shooting the Sun. Ritual and meaning in West Sepik que cette démarche prend forme de façon rigoureuse et surtout systématique. Ce livre présente une première interprétation des significations du rituel Yangis des Yafar - thème qui sera abondamment développé dans L'avènement $d u$ père - mais, surtout, il la confronte avec l'interprétation qu'Alfred Gell donne de Ida, le rituel Umeda correspondant à Yangis. Après le débat avec Ron Brunton et Alfred Gell dans les colonnes de Man en 1980, ce livre reprend de façon beaucoup plus substantielle et précise les écarts (i) entre Ida et Yangis, (ii) entre les approches anthropologiques d'Alfred Gell et de Bernard Juillerat. La confrontation, apaisée, de deux des anthropologues les plus créatifs de leur génération vaut à elle seule le détour. Mais le livre comporte aussi une série de chapitres écrits par des psychanalystes - André Green, François Manenti - et des anthropologues de premier plan - Marilyn Strathern, Roy Wagner, Richard Werbner, Donald Tuzin, Andrew Strathern - ce qui lui donne une place à part dans les publications océanistes des dernières décennies. À travers l'accent mis sur les significations des rituels et sur la pertinence de l'approche psychanalytique, on retrouve l'intérêt de Juillerat pour le langage, un langage dont il montre le dialogue constant avec des formes d'expression non verbales. En effet, l'étude du rituel Yangis amène Juillerat à développer un questionnement approfondi autour des significations non verbales, dans les actes et les objets en particulier. Là, d'autres nœuds se resserrent avec la psychanalyse :

« comme je l'ai suggéré, la motivation du rite est à la fois dans l'actuation et dans le sens, ou plus exactement dans l'actuation du sens. André Green se réfère souvent à Winnicott et j'ai aussi tenté de recourir à la notion d'espace potentiel à propos du rite. En ethnologie, il convient cependant d'apporter une mise au point. L'espace rituel est un lieu transitionnel entre le dedans et le dehors (Winnicott, 1975 : 146), participant de l'idée de limite définie par André Green, mais il tient davantage du game en ce que son déroulement est connu d'avance, fixé par la culture, tout en réservant une part de play, de créativité par l'expérience psychique renouvelée (individuelle et collective) vécue par les acteurs. À la fois dedans et dehors, le rite introduit dans son espace-temps transitionnel des objets empruntés au monde extérieur (plantes magiques, objets de culte) pour qu'il y signifient quelque chose du domaine tant culturel qu'intra-psychique, puis il les en écarte à nouveau. Ces objets matériels à chaque fois (re)trouvés sont intégrés aux règles du jeu, mais leurs mises en acte successives en font à chaque fois des objets (ré)inventés. » (2002 : 47)
Sous cet éclairage, le rite est conçu comme un contexte spécifique où les significations, des objets en particulier, effectuent des va-etvient entre personnes, culture et société. Ici encore, on remarque la place centrale donnée aux formes d'inscription du sujet dans la société et la culture.

Les ouvrages d'anthropologie psychanalytique de Juillerat sont d'une grande clarté. La qualité de leur argumentation contribue largement à leur force de conviction. Leur valeur ethnographique n'est pas moindre et c'est là le fil le plus constant de son œuvre. Mais cette problématique dominante de la dernière partie de l'œuvre ne doit pas faire oublier les autres thèmes abordés par Juillerat.

\section{Un anthropologue historien, mais hors des modes et des dogmatismes}

Par son refus de céder aux modes, Bernard Juillerat tient également une place à part dans le paysage des recherches sur la Mélanésie au cours des quarante dernières années. Par exemple, il est resté discret sur le statut de l'observateur ou la New Melanesian Ethnography, s'en tenant, si l'on ose dire, à une approche monographique classique des Yafar, avec le talent et les résultats que l'on sait. Il est de même resté à distance des oukases sur la subjectivité de l'anthropologue et n'a guère utilisé la notion de partible person. Il a souvent égratigné le structuralisme et on a vu qu'il a condamné l'anthropologie cognitive. Il est cependant essentiel de noter que, dans tous les cas, ce ne fut ni par désintérêt ni du fait d'une condamnation a priori que Bernard Juillerat ne s'est pas investi dans ces thèmes, pourtant bien présents dans l'anthropologie du moment. Sa position a toujours été mûrement réfléchie et, à cet égard, la façon dont il a traité l'histoire nous paraît exemplaire de la façon dont il a pu aborder un sujet sans se conformer aux injonctions comminatoires de certains spécialistes des sciences sociales.

Bernard Juillerat a parlé de l'histoire dans au moins quatre registres : à propos de sa part dans les transformations économiques et sociales des sociétés de Nouvelle-Guinée avant le contact (in Godelier et Strathern, 1991) ; dans sa réévaluation des travaux de Richard Thurnwald chez les Bánaro; dans son approche des bouleversements intervenus chez les Yafar depuis leur découverte des Blancs; et à propos des rapports entre histoire et anthropologie en général, à l'occasion des débats ouverts par l'" anthropologie historique de l'Océanie ». 
Comme d'autres chercheurs, Bernard Juillerat a utilisé l'opposition entre "Big men» et "Grands hommes» proposée par Maurice Godelier et Marylin Strathern dans Big Men and Great Men: Personifications of power in Melanesia (1991) pour la contraster avec une configuration politico-économique n'entrant dans aucune de ces deux catégories, ici celle des Yafar. Dans «Complementarity and rivalry: two contradictory principles in Yafar society » (1991), il décrit et analyse un cas intermédiaire entre les deux pôles dans lequel les experts rituels ne sont ni des Big men ni des Grands hommes - notamment du fait de la coexistence chez les Yafar de charges rituelles héréditaires et de statut acquis comme celui des médiums -, mais tiennent néanmoins de l'un de ces statuts d'hommes « influents» que l'anthropologie de la région peine à cerner.

Appliqué aux Yafar, le terme Big man est volontairement abusif et, si Bernard Juillerat l'accole à des personnages ne manipulant aucune richesse matérielle, c'est pour expliquer que des formes de rivalité, de pouvoir "achieved» peuvent se mettre en place à propos de situations dans lesquelles la manipulation de ces richesses ne joue aucun rôle. En l'occurrence, des formes de rivalité et de lutte pour le prestige entre des hommes éminents (initiateur de fission de hameau, responsable de culte de type millénariste) qui jouent sur des contradictions sociales pour se distinguer. On est loin des « systèmes de Big men » ou des cycles d'initiations complexes, « socialement plus élaborés » (1991: 141), mais il y a néanmoins lieu de s'interroger sur les transformations sociales conduisant, hors de toute manipulation de richesses, à la détention d'une ébauche d'autorité susceptible de passer de la sphère rituelle à la sphère politique par un effet cumulatif (1991 : 132-133). Chez les Yafar, il existe une complémentarité entre les charges qui reviennent à quelques-uns - expert rituel, leader politique, responsabilité dans le culte du cargo Wes apparu chez eux en 1981. Mais, au sein de chacune de ces sortes de spécialités, les hommes rivalisent entre eux, sans que l'on soit pour autant dans un système à "Big men" ni à « Grands hommes » (1991: 140).

C'est dans ce contexte, par rapport à l'histoire et à l'ethnographie des Yafar, que Bernard Juillerat s'est refusé d'interpréter la mise en place d'une ébauche de pouvoir et des stratégies qui l'entourent et, plus largement, de processus de complexification sociale, comme le seul résultat de différences structurales - comme l'opposition soulignée par Maurice Godelier autour de ces deux grands types d'institution sociale pesant lourdement sur la vie collective que sont les initiations masculines et les grands échanges cérémoniels de richesses. Pour lui, tant un système élaboré d'initiations masculines qu'un ensemble d'échanges cérémoniels étaient peut-être les résultats de processus où l'histoire joue son rôle. En l'occurrence, un rite comme Yangis (qu'il qualifie de «pseudo-initiation ») aurait présenté peu de possibilités de transformation en des initiations masculines (1991: 141).

Bernard Juillerat fut également historien des disciplines et des recherches scientifiques. Par ses écrits sur les rapports entre psychanalyse et anthropologie déjà évoqués, d'abord, mais aussi sur l'histoire de l'anthropologie de la Mélanésie, avec la recherche mêlant enquête de terrain et travail sur archives qu'il a mené à propos de Richard Thurnwald. Rappelons simplement que Bernard Juillerat choisit d'aller chez les Bánaro à la fois parce qu'il s'agissait a priori d'une société du Sépik fort différente des Yafar, et parce que nul n'y était retourné enquêter depuis 1915, malgré la complexité décrite par l'ethnologue allemand - notamment un système de parenté à moitiés, des rites d'initiation (masculine et féminine) associés à des échanges institutionnalisés de partenaires sexuels. L'enquête de terrain de Bernard Juillerat devait montrer que 75 ans de contacts coloniaux et missionnaires avaient suffi à détruire la société bánaro ancienne jusqu'à en effacer le souvenir de la plupart des mémoires, mais, tirant le maximum de la mémoire des informateurs et de sa confrontation du modèle de Thurnwald avec sa propre enquête, c'est une ethnographie contemporaine des Bánaro qu'il a esquissée dans $L a$ révocation des Tambaran (1993) faisant une large place à l'ethnohistoire. C'est l'occasion d'une mise en cause de l'ethnographie pratiquée loin du terrain, avec des informateurs peu fiables - et guère consentants dans le cas Bánaro -, et de remarques sur l'impatience théorique de Richard Thurnwald. Il s'est également attaché à replacer la recherche de Thurnwald, l'un des plus grands ethnologues de son temps, dans la tradition intellectuelle particulière au sein de laquelle il a fait œuvre originale, y compris en adoptant une démarche fonctionnaliste qui faisait une large place aux facteurs historiques.

L'irruption de la modernité chez les Yafar est un autre domaine où l'aisance de Bernard Juillerat à travailler simultanément sur des sources historiques et à partir de ses enquêtes de terrain fit merveille. Consacrées aux transformations rapides de la vie des Yafar au fur et à mesure de ses cinq séjours, entre juin 1970 et fin 1986. Les pages de «My poor border dwellers » (1997; 
également publiées en anglais dans Oceania [1993 : 59-88]), sont parmi les plus belles et les plus fines que compte la littérature ethnologique. Tout y est : la description précise des diverses manifestations matérielles de la modernité, la complexité d'une société qui se délite et même quelques lignes de ce regard de l'ethnologue sur lui-même dont Bernard Juillerat a dénoncé les excès (2001 : 9-10). Il rend ainsi compte de la distante présence missionnaire, de la vie des hommes yafar dans les plantations de coprah et de l'émerveillement de ceux restés au pays devant ce qu'ils en rapportèrent : sandales, t-shirts, guitare et une nouvelle langue, le Tok Pisin. Il parle de la construction de la route désenclavant le territoire yafar ou de la découverte par les Yafar du ravitaillement des soldats australiens de passage. Il raconte l'arrivée des lointains échos de la démocratie au moment de la préparation de l'Indépendance, l'introduction d'un fusil de chasse, la visite d'anciens ennemis, l'école, la fin du culte Yangis, le dépôt de secrets dans les carnets de l'enquêteur, les balbutiements des cultures de rente. Dans cet article aussi bref que détaillé, il relate aussi les épidémies, l'embrigadement des Yafar et les promesses de résurrection des morts dans le culte millénariste Wes, la mort de May Promp - l'incomparable « ami, informateur et contradicteur» (1986: 15) -, et même les inquiétudes de l'anthropologue et sa tristesse devant un monde bouleversé et menacé : au début des années 1990, "Yafar ne répondait plus» (1997: 81). En vérité, on ne saurait résumer la richesse de cet article qui, à lui seul, condense les qualités d'ethnographe de Bernard Juillerat, la finesse de ses analyses de phénomènes complexes et sa sensibilité d'homme de terrain - avec ce mélange d'amitié, d'angoisse et de responsabilités que chacun reconnaîtra.

Fait remarquable, on ne trouvera dans ces pages aucune référence à l'« invention de la tradition » ou à la «manipulation des agents de la modernité » et tout juste une allusion aux problématiques que les tenants de l'" anthropologie historique de l'Océanie » exigèrent un temps de voir partout traiter par les anthropologues, comme la vie urbaine, les migrations ou la politique électorale (par exemple Carrier 1992: 7). Sur ce point comme sur d'autres (l'anthropologie cognitive, par exemple), Bernard Juillerat associa une prise en compte du problème en question, ici, la modernité, à une critique très argumentée et virulente des positions extrémistes du moment.
Publiée sous la forme d'un « À propos » dans L'Homme, « L'histoire en morceaux » (1999) fut l'une des rares réactions aux excès et au dogmatisme du livre de Nicholas Thomas, Hors $d u$ temps (1998), au moins en français (sur ce point, voir Lemonnier, 1999). Après avoir résumé les arguments du livre et souligné les difficultés de Nicholas Thomas à définir des changements sans « temporaliser les variantes entre cultures » (1999 : 168-167), Bernard Juillerat a successivement rappelé l'impossibilité de faire " une histoire à long terme pour des sociétés qui ne connaissent pas l'écriture » (1999: 168); l'erreur de prendre l'irruption des Européens comme un « changement comme les autres » qu'il est possible d'" inscrire dans la continuité de l'histoire précoloniale » alors qu'il s'agit «d'un raz-demarée planétaire qui restera un fait unique dans l'histoire de l'humanité » (1999: 169); enfin et surtout, Bernard Juillerat reprochait à Thomas ce "paradoxe» qui « consiste à se convaincre que les études synchroniques de systèmes sociaux sont secondaires par rapport aux approches historiques, sans comprendre que celles-ci se fondent sur des études synchroniques d'autrefois » $(1999: 170)$.

Répondant avec agacement et fermeté aux oukases de Thomas - qui rejoignent ici ceux d'Alban Bensa (1996), Aletta Biersack (1991) ou James Carrier (1992) -, Bernard Juillerat, homme de terrain s'il en fut, dénonce le « procès d'intention » (1999: 170) de l'auteur de Hors du temps envers l'ethnographie et l'analyse anthropologique. Il rappelle le désarroi, « les frustrations » et les efforts de l'ethnographe pour remonter le passé, là où n'existe aucune source historique permettant de le faire. Visant directement la tentative de Nicholas Thomas de rendre compte de l'histoire de la Polynésie dans la longue durée, Bernard Juillerat se demandait s'il ne «vaut pas mieux analyser (les) réponses à l'influence occidentale saisies sur le vif que de se risquer à des reconstitutions hasardeuses sur les grandes étapes évolutives des systèmes sociaux ... ?» (1999: 170). Rejoignant LéviStrauss (sans le vouloir?), il plaidait pour des recherches parallèles « laissant chacun, anthropologue et historien, faire son travail » (1999: $170)^{3}$. Bernard Juillerat plaide pour une observation de la durée dans le présent du terrain ethnographique :

«C'est le temps de l'observation par excellence, où l'histoire se fait sous nos yeux, dans le laps de temps d'une vie d'ethnologue. » (1999: 171) 


\section{Présentation des articles}

Ce numéro spécial du Journal de la Société des Océanistes en hommage à notre regretté collègue Bernard Juillerat s'ouvre avec deux textes évoquant des collaborations scientifiques de ce dernier. Dans "Remembering Bernard Juillerat. Visiting the Bánaro after Richard Thurnwald », Marion Melk-Koch raconte ses échanges avec Juillerat à propos des travaux de Richard Thurnwald et, en particulier, de ses notes de terrain qui, en raison des nombreux voyages - parfois forcés - de l'anthropologue berlinois, sont dispersées dans le monde entier. Juillerat a en effet revisité les Bánaro de Papouasie NouvelleGuinée dans La révocation des Tambaran (1993). Il a préparé son terrain par des recherches des sources anciennes à Berlin, ce qui fut l'origine d'échanges d'abord épistolaires, puis de deux rencontres personnelles entre Marion et Bernard. Ce texte évoque les péripéties de la recherche des archives anthropologiques et les surprises réservées par une société, effleurée par Thurnwald en 1913 et revisitée par Juillerat en 1989 et 1990.

Dans « Lettres à un jeune ethnologue », Philippe Peltier publie quatre lettres tirées d'un échange de correspondance avec Juillerat pendant son second terrain dans le Sépik. Chercheur expérimenté, celui-ci réagit aux notes de terrain que lui a confiées Peltier. Née d'un désir de donner à entendre à nouveau la voix de Bernard Juillerat, cette publication d'un autre aspect de son activité épistolaire nous renseigne aussi bien sur sa manière de mener les entretiens que sur son appréciation du milieu ethnologique parisien ainsi que sur de multiples choses encore, petites ou grandes, qui font les échanges entre collègues engagés sur un terrain proche.

Avant de devenir spécialiste de l'Océanie, Juillerat avait travaillé au Cameroun, chez les Mouktélé, où il fut l'un des pionniers de l'ethnologie dans la région reculée des Monts Mandara. Le contexte de ses travaux dans cette partie de l'Afrique nous est présenté par sa collègue Jeanne-Françoise Vincent dans un article qui présente de façon détaillée et souvent comparatiste les descriptions et analyses du jeune Juillerat dont les recherches africanistes se caractérisaient déjà par leur minutie ethnographique et leur honnêteté intellectuelle.

D'août à novembre 2008, le Musée de Port Moresby a présenté une exposition intitulée $A$ tribute to Bernard Juillerat: The Iafar between symbols and artifacts. Une partie de la collection rassemblée par Bernard Juillerat et donnée au musée en 1974 y était présentée au public. Cette exposition, qui montre le rayonnement du travail de Juillerat dans le pays où il effectua ses terrains océaniens, est présentée ici de façon détaillée par Nicolas Garnier et sert de point de départ à une réflexion sur le rôle d'un musée comme celui de Port Moresby. Il est au confluent de la culture que les habitants de Papouasie Nouvelle-Guinée vivent au quotidien ou lors d'événements plus ritualisés et de celle que leur renvoient les institutions émanant de l'influence occidentale, dont l'anthropologie et la muséographie. Doit-il se concentrer sur une mission de conservation des objets ou bien se consacrer aussi au montage d'expositions thématiques temporaires telle que A tribute to Bernard Juillerat ? À la diversité des interrogations répond celle du public, comme le montrent les réflexions de deux des visiteurs de cet hommage muséographique.

Le texte d'André Green complète les précédents en montrant la diversité et la richesse de l'homme Bernard Juillerat en son œuvre, tout en introduisant pour ce volume la perspective psychanalytique qui marque profondément la dernière partie de celle-ci. De leur dialogue et de leur collaboration, il évoque le déroulement, depuis la participation du psychanalyste à la recherche collective de Shooting the sun (1992). Green énumère les thématiques principales développées par Juillerat. Ainsi, la démarche psychanalytique remet profondément en cause certains « dogmes » structuralistes pour ouvrir sur des prises de position radicales, comme celle qui affirme que "le sens détermine la structure $»$.

Jadran Mimica présente une étude de cas ethnographique traitée de façon psychanalytique, celle d'un garçon yagwoia-anga de Papouasie Nouvelle-Guinée. La dialectique de la paternité yagwoia met en ouvre une configuration dynamique conditionnée par le désir de la mort du père, celle-ci étant la condition du transfert et de la continuation dans les fils de la "puissanceosseuse » (bone-power). Les circulations, d'origines paternelle et fraternelle, de puissanceosseuse, nourritures, biens affinaux et autres étant détournées et refusées à ce garçon dont la position générationnelle bien spécifique se caractérise par une incomplétude relationnelle, Mimica montre comment il rompt avec le côté paternel de sa parenté. Le garçon se réfère alors prioritairement au côté maternel et à de la poudre d'os humains achetée à un étranger. Ceci se manifeste à travers des rêves qui associent d'une part son nourrissage et sa croissance à un oncle défunt et sa force guerrière et d'autre part son éloignement de la société à cette poudre d'os qu'il conserve sur lui. 
Dans « Kinship, Ritual, Cosmos », Pamela Stewart et Andrew Strathern confrontent deux usages des théories psychanalytiques en anthropologie, celle de Meyer Fortes et celle de Bernard Juillerat. Tous deux font usage de la théorie de l'Edipe, mais en privilégiant certains aspects qui correspondent à leurs ethnographies respectives (voir aussi Douaire-Marsaudon ci-dessous). Les Yafar mettent l'accent sur les relations mère-fils et leur nécessaire disjonction pour que les garçons deviennent des hommes - un thème assez répandu en Nouvelle-Guinée. En contraste, pour les Tallensi d'Afrique de l'Ouest, Meyer Fortes privilégie les rapports du père et du fils aîné. Chaque auteur, chaque peuple, privilégie un côté du triangle œdipien. Alors que ces configurations œdipiennes sont à la marge de l'ethnographie, celles-ci occupent une place centrale dans les interprétations des deux auteurs.

Françoise Douaire-Marsaudon présente une lecture de mythologies et de rites polynésiens dans la perspective du «drame éclaté de l'Edipe » (Bernard Juillerat), vu ici dans une autre configuration de fusion originaire, de retour au stade intra-utérin. Des questions tournant autour de l'inceste, de la césure nature/culture et de la place éminente de l'engendrement et de la production des générations sont reprises ici à travers le matériau polynésien. À considérer les oscillations entre le Po-nuit et le 'Aho-jour, on peut dégager un élément-clé, véritable interface entre les deux domaines, la notion polymorphe de fonua qui signifie à la fois « terre/pays », « tombe » et « placenta ».

L'interprétation des rêves fut un des thèmes centraux du travail de Freud et nombre d'ethnologues en ont montré l'importance dans d'autres civilisations. Chez les Kanak de NouvelleCalédonie, les rêves sont souvent évoqués dans les conversations, leur importance a été soulignée par certains observateurs, mais ils n'ont pas été objets d'études fouillées. Isabelle Leblic les met en contexte pour dégager les lignes de force de leur prégnance sociale et culturelle. Dans un monde où «créations » et «innovations » sont souvent considérées comme inspirées par les ancêtres et/ou les esprits, le rêve est une passerelle, moyen privilégié d'accéder à des savoirs qui doivent être authentifiés ou n'ont pas été transmis. Ainsi, invention du masque, quête de médicaments, représentations picturales, chants et danses mais aussi recherche universitaire peuvent-ils être référés à l'activité onirique. Le rêve, enfin, permet la communication avec des parents ou amis vivants qui se sont éloignés, il est parfois porteur, ou même précurseur, de nouvelles les concernant, ou ayant trait à la marche du monde. Leur décryptage met en œuvre une symbolique qui partage des traits significatifs avec la littérature orale.

Marilyn Strathern interroge les notions de don et de réciprocité. Juillerat a en effet proposé pour les Yafar de « détacher » l'idée de réciprocité de celle du don, se positionnant clairement contre des positions centrales chez Mauss et Lévi-Strauss. Déplaçant le regard anthropologique de la Mélanésie vers le monde occidental, Marilyn Strathern, après avoir rappelé l'influence de l'Essai sur le don dans certaines théories récentes concernant le don du sang montre, pour ce dernier et aussi pour le don d'organes, l'existence de débats à propos de questions connexes à la réciprocité. Le don du sang ou d'organes serait altruiste, volontaire, « don de vie », toutes qualifications morales qui semblent s'opposer à la marchandisation alors même qu'un auteur souligne que le don d'organe commence sa trajectoire de façon altruiste pour l'achever dans le commerce. Il existe aussi des différences significatives selon le type d'organe concerné. Toutefois il semble que la notion de don, elle, ne soit guère remise en cause.

L'anthropologie des sentiments et en particulier celle du bonheur restent peu développées dans la recherche française contemporaine. Monique Jeudy-Ballini montre que chez les Sulka de Papouasie Nouvelle-Guinée, tandis que l'expression des émotions est relativement libre, il est inconvenant de spéculer sur les raisons de ces émotions chez autrui. Il y a donc une réelle difficulté à faire l'anthropologie des sentiments, ce qui rend la tâche d'autant plus stimulante. En effet, comprendre une société, c'est connaître les manières de sentir de ses membres, savoir comment elles informent ou organisent leurs interactions sociales, et de quelle façon elles peuvent constituer des modes d'action, ce qu'avait bien compris l'école «culturaliste» américaine. Un tour d'horizon des approches récentes de la question des émotions et de l'affectivité, enrichi par des allers-retours avec l'étude des Sulka, suggère comment la prise en compte des émotions pourrait contribuer à fédérer anciens et nouveaux thèmes et terrains de l'anthropologie.

Gilles Bounoure fait le compte rendu des deux derniers ouvrages de Bernard Juillerat, Penser l'imaginaire. Essais d'anthropologie psychanalytique (2001) et le collectif édité avec Patrice Bidou et Jacques Galinier, Psychanalyse et anthropologie. Regards croisés (2005). Bounoure met en valeur la position centrale de la psychanalyse dans l'œuvre de Juillerat, les croisements qu'il propose avec l'ethnographie et la place des découvertes de Freud dans des débats intellec- 
tuels qui, comme le montre l'actualité récente, n’ont jamais cessé d'être passionnés et passionnants.

Sophie Chave-Dartoen place l'ethnobotanique au cœur de sa réflexion pour mieux éclairer les relations sociales. Elle reprend le problème des représentations et de leur efficacité à partir de la «production des jardins » (Juillerat), mais pour le terrain polynésien de Wallis. En effet, l'importance rituelle des plantes cultivées, des techniques et des pratiques qui leur sont associées ne peut être pleinement saisie à travers une opposition entre matériel et symbolique. Les ignames sont la prestation masculine par excellence et leurs récoltes sont considérées comme une manifestation de relations bénéfiques entre le pays - aux premiers rangs duquel les horticulteurs et les anciens -, le roi et Dieu.

Christian Coiffier s'intéresse à l'utilisation, dans de nombreux rituels, de plantes productrices de fruits dont la couleur change avec la maturation. Bernard Juillerat a essayé de comprendre de façon rigoureuse la perception profonde de certains de ces fruits pour ses informateurs Yafar. Élargissant la perspective à plusieurs sociétés du Sépik, Coiffier remarque que, souvent, ces plantes renvoient à la mort ou au vieillissement humains et que sont privilégiées celles d'entre elles considérées comme étant en relation avec le monde chtonien.

Les trois dernières contributions, si elles font écho à la thématique du sujet et des relations, s'intéressent plus particulièrement aux significations portées par les cérémonies et les rituels. En écho avec l'interprétation de Juillerat selon laquelle l'accomplissement du rituel met en relief des valeurs sous-jacentes aux relations sociales, Christian Kaufmann se penche sur le rôle dévolu par les Iatmul de Papouasie Nouvelle-Guinée à des objets marquant des relations de genre. Dans des rituels comme le naven, des artefacts objectifient - rendent visible - ce type de relations. Même s'ils ne circulent pas, ils peuvent être considérés comme des "dons » de savoir et de service, car précisément ce qui circule dans ces circonstances est l'expression du savoir par la monstration et non la circulation des objets.

À partir de cérémonies kanak de NouvelleCalédonie, Denis Monnerie propose de distinguer entre «symboles » et «figures ». Ces dernières - comme la Maison ou Grande Maison renvoient toujours à un même thème qui, dans les cérémonies, est mis en œuvre sous des formes qui visent à associer systématiquement compréhension et expériences plurisensorielles et à le faire de façon collective. Les formes minimales de cette mise en œuvre cérémonielle collective du social pour les figures et leurs significations sont des paires, ce qui renvoie aux « fantasmes originaires » privilégiés par Juillerat, qui, tous, mettent en jeu des relations entre au moins deux acteurs.

C'est à une réflexion comparatiste sur les mythes et les rites des peuples anga de Papouasie Nouvelle-Guinée que nous convie Pierre Lemonnier, pour mieux y comprendre la place du sujet. S'il n'y a pas de «nécessité dans la logique d'un imaginaire collectif », il y a des conventions. Comment celles-ci font-elles partie des sujets ? La place des rituels est ici centrale. Les transformations que le sujet masculin subit lors des rituels d'initiation ankave débordent le seul moment rituel car il y ressent, de façon verbale et non verbale, des actions, des émotions et des savoirs relatifs au monde où il est né et dans lequel il vit.

\section{BIBLIOGRAPHIE}

BARTH Frederik, 1975. Ritual and Knowledge among the Baktaman of New Guinea, Oslo, Universitetforlaget, New Haven, Yale University Press.

-, 1987. Cosmologies in the Making. A Generative Approach to Cultural Variation in Inner New Guinea, Cambridge, Cambridge University Press.

BATESON Gregory, 1971 ( $1^{\mathrm{e}}$ éd. 1936). La cérémonie $d u$ Naven. Les problèmes posés par la description sous trois rapports d'une tribu de Nouvelle-Guinée, Paris, Éditions de Minuit.

BENSA Alban, 1996. De la micro-histoire vers une anthropologie critique, in Jacques Revel (éd.), Jeux d'échelles. La micro-analyse à l'expérience, Paris, Gallimard, Le Seuil, Hautes études, pp. 37-70.

BIERSACK Aletta, 1991. Clio in Oceania. Toward a historical anthropology, Washington, Londres, Smithsonian Institution Press.

BRUnton Ron, 1980a. Misconstrued order in Melanesian religion, Man 15 (1), pp. 112-28.

_, 1980b. Order or disorder in Melanesian religion? Correspondence, Man 15 (4), pp. 734-735.

CARrier James (ed.),1992. History and tradition in Melanesian anthropology, Berkeley, Los Angeles, Oxford, University of California Press.

Dadoun Roger, 1967. Avant-propos : présentation de Géza Ròheim, in Géza Ròheim, Psychanalyse et anthropologie, Paris, Gallimard, pp. 9-22.

DAMON Frederick and Roy WAGNER (eds), 1989. Death Rituals and Life in the Societies of the Kula Ring, DeKalb, Northern Illinois University Press.

FELD Steven, 1979. Sound and Sentiment. Birds, Weeping, Poetics, and Song in Kaluli Expression, Philadelphia, University of Pennsylvania Press. 
Gell Alfred, 1975. Metamorphosis of the Cassowaries. Umeda Society, Language and Ritual, London, The Athlone Press.

- 1980 . Order or disorder in Melanesian religion? Correspondence, Man 15 (4), pp. 735-37.

GODELIER Maurice, 1982. La production des grands hommes. Pouvoir et domination masculine chez les Baruya de Nouvelle-Guinée, Paris, Fayard.

Godelier Maurice et Marilyn Strathern, 1991. Big Men and Great Men. Personifications of power in Melanesia, Cambridge, Paris, Cambridge University Press-Éditions de la maison des sciences de l'homme.

Herdt Gilbert, 1981. Guardians of the Flutes. Idioms of Masculinity. A study of ritualized homosexual behavior, New York/St. Louis/San Francisco, McGraw-Hill Book Company.

_, 1987. The Sambia: ritual and gender in New Guinea, New York, Holt, Rinehart and Winston.

—, 2003. Secrecy and Cultural Reality: Utopian Ideologies of The New Guinea Men's House, Ann Arbor, The University of Michigan Press.

Houseman Michael et Carlo Severi, 1994. Naven ou le donner à voir. Essai d'interprétation de l'action rituelle, Paris, CNRS Éditions, Éditions de la maison des sciences de l'homme.

Humphrey Caroline et James LaIDlaw, 1994. The Archetypal Actions of Ritual: A Theory of Ritual Illustrated by the Jain Cult of Worship, Oxford, Clarendon Press.

Jeudy-Ballini Monique et Bernard Juillerat (éds), 2002. People and Things. Social Mediations in Oceania, Durham (N.C.), Carolina Academic Press.

JuILlerat Bernard, 1971. Les bases de l'organisation sociale chez les Mouktélé (Nord-Cameroun). Structures lignagères et mariage, Paris, Institut d'ethnologie, musée de l'Homme.

_, 1975a. Transe et langage en Nouvelle-Guinée ( $1^{\text {ère }}$ partie). I. La possession médiumnique chez les Amanab, Journal de la Société des Océanistes 31, 47, pp. 187-212.

_, 1975b. Transe et langage en Nouvelle-Guinée ( $2^{\text {ème }}$ partie). II. Du symptôme au rite, Journal de la Société des Océanistes 31, 49, pp. 379-397.

- 1980 . Order or disorder in Melanesian religion? Correspondence, Man 15 (4), pp. 732-34.

_, 1986. Les Enfants du sang : société, reproduction et imaginaire en Nouvelle-Guinée, Paris, Editions de la maison des sciences de l'homme.

_, 1992. Shooting the sun: ritual and meaning in West Sepik, Washington London, Smithsonian series in ethnographic inquiry, Smithsonian Institution Press, in cooperation with the ministère des Affaires étrangères (Paris).

—, 1993. La révocation des Tambaran : les Bánaro et Richard Thurnwald revisités, Paris, CNRS éditions, CNRS ethnologie.
-, 1995. L'avènement du père : rite, représentation, fantasme dans un culte mélanésien, Paris, CNRs Éditions, Éditions de la maison des sciences de l'homme, Chemins de l'ethnologie.

_, 2002. Le rite comme acte et comme représentation: de l'inconscient à l'espace transitionnel, in César Botella (éd.), Penser les limites : écrits en l'honneur d'André Green, Paris, Delachaux et Niestlé, pp. 44-51.

_, 2001. Penser l'imaginaire: essais d'anthropologie psychanalytique, Lausane, éditions Payot.

_, 2004. Psyche, culture and society: book review forum on Bernard Juillerat's « Penser l'imaginaire. Essais d'anthropologie psychanalytique », Journal of ritual studies 18, 1, pp. 129-166.

Juillerat Bernard, Patrice Bidou et Jacques GaliNIER (éds), 2005. Anthropologie et psychanalyse. Regards croisés, Paris, éditions de l'EHESS.

Lawrence Peter, 1964. Road Belong Cargo: A Study of the Cargo Movement in the Southern Madang District, New Guinea, Manchester, Manchester University Press.

LEMONNIER Pierre, 1999. La chasse à l'authentique. Histoire d'un âge de pierre hors contexte, Terrain 33, pp. 93-110.

LÉvi-Strauss Claude, 1962a. La pensée sauvage, Paris, Plon.

—, 1962b. La notion de structure en ethnologie, in Roger Bastide (éd.), Sens et usages du terme Structure, 'S-Gravenhage, Mouton.

LEWIS Gilbert, 1980. Day of Shining Red. An Essay on Understanding Ritual, Cambridge, Cambridge University Press.

Mosko Mark, 1983. Conception, De-conception and Social Structure in Bush Mekeo Culture, Mankind 14, pp. 24-32.

SCHIEFFELIN Edward Lawrence, 1976. The Sorrow of the Lonely and the Burning of the Dancers, New York, San. Martin's Press.

TuZIN Donald Francis, 1980. The Voice of The Tambaran. Truth And Illusion in Ilahita Arapesh Religion, Berkeley, University of California Press.

-, 1987. The Cassowary's Revenge: The Life and Death of Masculinity in a New Guinea Society, Chicago and London, The University of Chicago Press.

Silverman Eric Kline, 2001. Masculinity, Motherhood, and Mockery. Psychoanalyzing Culture and the Iatmul Naven Rite in New Guinea, Ann Arbor, The University of Michigan Press.

Thomas Nicholas, 1998. Hors du temps. Histoire et évolutionnisme dans le discours anthropologique, Paris, Belin.

Wagner Roy, 1973. Habu: The Innovation of Meaning in Daribi Religion, Chicago, The University of Chicago Press.

Whitehouse Harvey, 1995. Inside the Cult. Religious Innovation and Transmission in Papua New Guinea, Oxford, Clarendon Press. 
